\title{
Stress Recognition in Daily Work
}

\author{
Yoshiki Nakashima*, Jonghwa Kim**, Simon Flutura**, Andreas Seiderer**, \\ and Elisabeth André** \\ *Smart Energy Research Laboratories, NEC Corporation \\ 34 Miyukigaoka, Tsukuba, Ibaraki 305-8501, Japan \\ y-nakashima@bu.jp.nec.com \\ **Institute of Computer Science, University of Augsburg, \\ Universitätsstraße 6a, D-86159 Augsburg, Germany
}

\begin{abstract}
Automatic detection of work-related stress has attracted an increasing amount of attention from researchers from various disciplines and industries. An experiment is discussed in this paper that was designed to evaluate the efficacy of multimodal sensor measures that have often been used but not yet been systematically tested and compared with each other in previous work, such as pressure distribution sensor, physiological sensors, and an eye tracker. We used the Stroop test and information pick up task as the stressors. In the subject independent case in particular, signals from the combined (chair and floor) pressure distribution sensors, which we consider the most feasible sensors in the office environment, resulted in higher recognition accuracy rates than the physiological or eye tracker signals for the two stressors.
\end{abstract}

Keywords: stress, activity recognition, machine learning, multi-modality

\section{Introduction}

Stress is the wear and tear that our minds and bodies experience as we attempt to cope with our continually changing environment. In particular, stress at work can be very expensive. It is identified as the second most frequently reported work-related health problem in the world and believed to be the cause of more than half of all lost working days.

The demands to office workers are sometimes too high and when they feel that they cannot handle all of the demands based on their capabilities, they become stressed [1]. However, if they have enough time to recover from their stressed state, they will less likely incur mental illness. So, it would be beneficial if the emotional states of office workers could constantly be monitored in order to detect their levels of stress, since this would make workers aware of their stress level and encourage them to have recovery time. Moreover, this would assist their employers in preventing them from demanding too much of them. For this purpose, the most important point is the feasibility of implementing a stress recognition system in the office environment.

Sometimes stress recognition means to differentiate between 'stressed' and 'relaxed' state, but office workers are normally concentrating on their work, and thus, 
are neither relaxed nor stressed. In other words, this is the state that people are in while doing a task without any stressors. So, we should add a new state, so-called 'concentrated' state, and be able to recognize between three states, which are 'relaxed', 'concentrated', and 'stressed'. If we are able to differentiate between these three states, we make an important step forward towards the automated recognition of stress states in daily work.

There have been various modalities that were proposed for stress recognition, including facial expression, speech expression (prosody), physiological signals, eye movements, and postures. Among them, using postures is significantly advantageous for long term daily use, especially in an office environment. First, they can be sensed non-intrusively [2] and unobtrusively, unlike physiological signals, which means the sensors themselves never make people stressed. Second, they can be sensed all the time while people are at work, unlike speech or eye movements. Most eye movement sensors can only detect eye gaze points which are inside the display. Third, postures can be analyzed at a relatively coarse level, in comparison to facial or speech expressions, which means they offer substantial benefits in terms of simplicity [2].

Pressure distribution sensor mats have garnered a lot of attention as sensor devices suitable for posture detection in office environments. They have also been tested as sensors for recognizing stress or emotions in publications [4], [7].

Stress and emotions are theoretically related to activities of the autonomic nervous system (ANS) [3]. ANS activities are largely involuntary and generally cannot be easily triggered by any conscious or intentional control. Therefore, physiological signals, which are the results of these ANS activities [3], are considered as reliable signals of stress and emotional activities. The same thing can also be said for some specific eye movements, such as fixation or saccades.

These three modalities are respectively interesting for long-term stress monitoring, in different reasons. These modalities have been used in previous work, but not yet been systematically tested and compared with each other. It is beneficial to know which modality or which combination of modalities is the best solution.

\section{Related Work}

In this chapter, we briefly review previous related work in terms of an argument about the stress states to be classified, stress stimuli, and the sensor modalities used for automatic detection.

Stress stimuli. A Stroop test, especially a computer-based one, has been evaluated as an effective stressor in previous work on automatic stress recognition [5], [6]. There are two Stroop test versions, 'congruent' and 'incongruent'. In the congruent test, a screen containing a color name like "yellow" which is inked in the same color is shown to the participant. In the incongruent test, a color name is also shown to the participant, but in this case it is inked in a different color. For example the name of the color is "green" but it is inked "red". In both tests, the participant should answer with the name of the color the word is inked with. In the incongruent test, she/he should answer with a different word than she/he is looking at. This leads to stress 
resulting only out of the conflict between the recognition of the word and the color, and thus, does not depend on the participant's ability like it would be the case if calculations are used, so all participants should be stressed at a nearly equal level.

More natural-like tasks that mimic typical office work have been used as mental stressors. For example, text transcriptions, information pick up tasks, and expressive writings were designed as mental stressors in [8]. The office work stressors are more appropriately being evoked in these tasks. For these tasks, the stressors are also designed to be typical for office work, such as time pressure and interruptions (by phone calls or E-mails). These stressors force mental workloads on the participant. One research [9] classified the workload factors that are common in many kinds of work into [10] classes, and showed that the most important factor is time pressure. Relaxing videos are typically used for inducing a relaxed state [8].

Modalities. Several studies showed that pressure distribution sensors are an effective modality for recognizing stress by recognizing the participant's posture [4], [7]. Typically, the pressure distribution on a person's seat is sensed, and then the postures are recognized from the sensor data. Finally, the emotional and stress states can be determined using these recognized postures. Nevertheless, recent research has shown that the pressure distribution data itself can be used to recognize stress, without needing to first recognize the postures [4]. Furthermore, floor pressure distribution can be used for emotion recognition [10]. Peripheral physiological signals [11], [12] have been proven to be effective stress or emotion recognition modalities. Electro dermal activity (EDA) is one of the most effective signals from among the physiological signals used for stress or emotion recognition [12]. Blood volume pressure (BVP) and heart rate (HR) are also preferred as stress or emotion recognition features [13]. Involuntary eye movements, like saccades or fixations have also been investigated and shown to reflect the activities of the central nervous system, and are related to stress and emotions [14]. So, eye movement signals, which can be tracked by eye tracker sensors, can also be added as an effective modality for stress recognition.

\section{Experimental Conditions}

Stress Stimuli. We used the Stroop test and the information pick up task as the stress stimuli. For the Stroop test, a congruent test is used for the concentrated state and an incongruent one is used for the stressed state. The only difference between them is whether there is a mismatch between the color and the word. There are six colors to select. The time limit for an answer is three seconds and the whole duration of each test is three minutes (Figure 1).

The participant reads an HTML page with around 900 words for the information pick up task. The participants are instructed to pick up 10 pieces of information from that page. There are two conditions. First is to induce a stressed state using a 3-minute time limit as time pressure, emphasized by a timer shown on the display. The second task is for the concentrated state without stressors. 


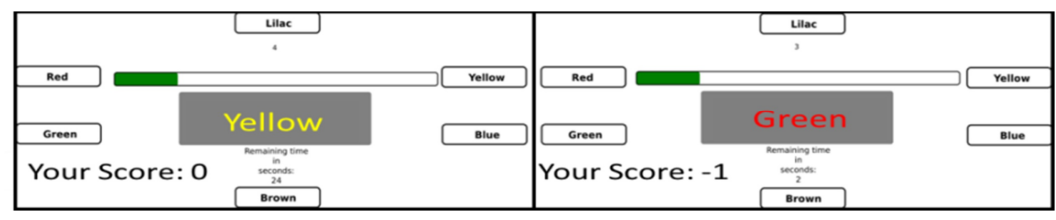

Figure 1 Congruent (left) and Incongruent (right) Stroop Tests.

Sensors. Our setup includes three pressure distribution sensors (on the floor and on the seat and backrest of a chair), an eye tracker sensor, and a physiological sensor. We used SensingTex [15] sensors for the pressure distribution sensors. The seat and backrest chair sensors have a $4.5-\mathrm{cm}$ resolution with $8 \times 8$ pressure detection cells, and the floor mat sensor is a $16 \times 14$ cells' sensor. The sampling rate for all of these SensingTex sensors was set to $20 \mathrm{~Hz}$. With these three pressure sensors (chair seat, chair backrest and floor), we are able to monitor the whole body movement of a person in a sitting posture. Making these three sensors coalesce with other modalities is, at least in our knowledge, the first attempt. We selected the IOM [16] sensor for the physiological data. The sampling rate was $27 \mathrm{~Hz}$. This sensor delivers BVP and EDA data. We can also obtain HR from the BVP signal by using signal processing. We used the Eye Tribe [17] as stationary eye tracker. Its sampling rate is $30 \mathrm{~Hz}$. The setup of these sensors and photographs of the setup are shown in Figure 2. The Social Signal Interpretation (SSI) framework was used for the synchronized recording of these multimodal sensor data [18].

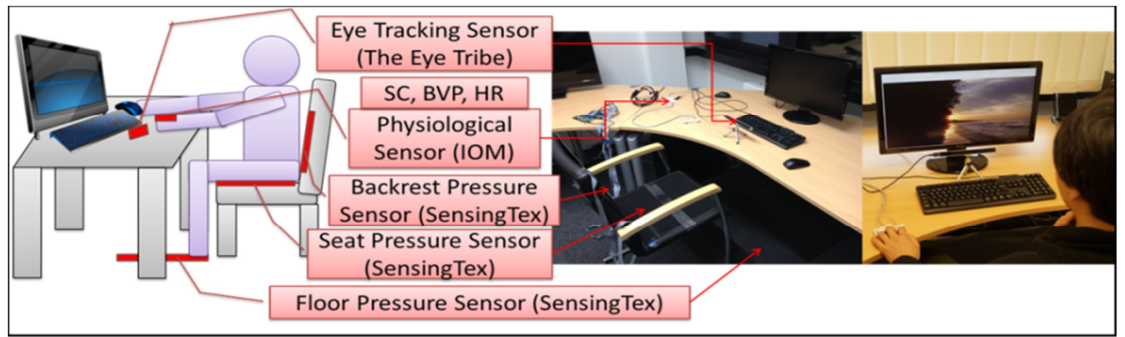

Figure 2 Sensors used in our study (left), and photographs of set-up (center) and a participant doing task in set-up (right).

Participants. Ten healthy volunteers participated in our experiment. There were two women and eight men with an average age of 31 years. All of them were right handed and used the right hand to operate the computer mouse.

Experimental Procedure. We divided the participants into two groups, and set different schedules for each. Before the experiment, the participant was informed orally and through a document about the purpose of the experiment and the procedure that should be regarded. Then, each of them was connected to the physiological sensors. The eye tracker calibration also took place at this time. After that, each participant answered the first questionnaire. The questionnaire included the age, gender, dominant hand, and the hand she/he usually uses to operate a computer mouse. Afterwards, the participant shortly practices the congruent and incongruent Stroop tests to make sure they are understood correctly. Finally the experiment starts. 
Figure 3 Time schedule for two groups. INC stands for the incongruent Stroop test, CON for the congruent Stroop test, TP for the information task with time pressure, and No TP for the information task without time pressure.

We begin with a 5 minute relaxation session to relax the participant and to record the data of the relaxed state of the participant. During this session a video of landscapes accompanied with chill out music is shown. Then, a NASA TLX (Task Load Index) questionnaire [9] follows. The questionnaire consists out of six questions, asking their level of mental demand, physical demand, temporal demand, performance, effort, and frustration. We omitted asking the physical demand among these questions, because our purpose is to recognize the stress of office workers, who generally do not do physically demanding work.

The session with the Stroop tests begins after the questionnaire. For the first group, the incongruent test begins first, followed by the congruent test. For the second group the order is reversed, and then a 2 minute relaxation video is shown followed by a NASA TLX questionnaire.

Then, the information tasks begin. A NASA TLX questionnaire follows each task. For the first group, the task with time pressure was done first. The second group starts with the task without time pressure. The 2 minute relaxation video follows after the proceeding one. Ten pieces of information have to be gathered from around 900 words of text during the information pick up task. The text's context is related to fruit. The texts consisted out of adapted Wikipedia articles and were written in the mother tongue of the participants. The number of words was decided based on the known fact that the average reading speed is around 200 to 300 words per minute [19]. So 3 minutes is barely enough time for average readers.

\section{$4 \quad$ Analysis}

As we previously discussed, we used pressure distribution sensors, the eye tracker and physiological sensors. We calculated the features explained below within the time duration of 10 seconds, which we define as our event duration. Since we set the time duration of each stimulus (Stroop tests and information task) to 3 minutes or 180 seconds, we received 18 events for each stimulus.

Pressure Sensor. For the pressure distribution sensors, we focused on detecting the "Center of Pressure" or the weighted average point of distributed pressure loads for these sensors' two-dimensional coordinates. The two dimensions are AP (anterior-posterior) and LR (left-right). We used the euclidean distance (ED) to introduce a combined feature. Additionally we included the total intensity (TI) of each pressure distribution sensor mat. We calculated the statistical features and frequency features for these four values. The statistical features are the average, standard deviation, first and second difference, and the normalized first and second difference [20]. The frequency features are several body sway frequency bands that were 
reported to be related with stress or emotions: $0.1-0.7 \mathrm{~Hz}, 0.7-1.3 \mathrm{~Hz}$ [21], and $3.5-8.0 \mathrm{~Hz}$ [22]. The experiments reported on in these literatures were conducted with the participants standing, but these characteristic frequency bands depend on the time duration of the internal neural control system [21], so we also decided to use these frequency features. We also added the $1.3-3.5 \mathrm{~Hz}$ frequency band, which is in between the above bands, to see if there would be some relationship between the stress and this adjoining band.

Physiological Sensor. We calculated six statistical values (averages, standard deviations, first and second differences, and normalized first and second differences) [20] and the peak response time, peak amplitude, and energy [25] for EDA. We used the mean amplitude, skewness, and kurtosis [26] and the six statistical values of the signal for the blood volume pulse (BVP). Finally, we calculated the RMSSD [27], very low $(0.05-0.15 \mathrm{~Hz})$ and low $(0.15-0.4 \mathrm{~Hz})$ frequency bands and their ratio (low/very low), and the six statistical values for HR.

Eye Tracker Sensor. We calculated blinks, fixations, saccades, and scans [23], [24] from the eye tracker signals. We obtained the time duration, space distance (excluding blinks), and the number of occurrence of these four eye states, and calculated the maximum, mean, and summation values for these time durations and space distances as our features.

\section{Classification}

Feature Selection. We first try to select the features for each modality that we use, which are EDA, BVP, HR, floor, backrest, and the seat pressure distributions, and the eye tracker. We also set the physiological combined modality, which is the combination of EDA, BVP, HR, and the pressure distribution combined modality, which is the combination of the floor, backrest, and seat pressure distributions. We used sequential backward selection (SBS) as the method for the feature selections. The criterion for the SBS was pLDA.

Modality Level Classification. After selecting the features of each modality, we calculated the recognition accuracy rate of each modality for each state, using pLDA (pseudo LDA). We call the classification result of each modality for each state a 'decision'. Along with these modality decisions, we have also calculated the classification accuracy rate using all the modalities as a reference.

Decision Level Classification. The decision level classification [28] refers to the classification by combining the 'decisions' from multiple modalities. We used the recognition accuracy information for each state using the features of each modality first, and then, multiplied the accuracy rates as the weights of each decision for each modality. When the prediction accuracy for state $j$ using the features extracted from modality $i$ is defined as $P_{i j}$, and the 'decision' for state $j$ using modality $i$ is defined as $X_{i j}$, then the weight decision for state $j$ can be expressed as $X_{j}$ in equation (1).

$$
X_{j}=\frac{\Sigma_{i} P_{i j} X_{i j}}{\Sigma_{i} P_{i j}} .
$$


These weighted decisions for each state can be the features, and we made use of these 'decision level' features to train the classifiers. We used five classifiers in this 'decision level classification' phase, which are kNN ( $k=1)$, pLDA, Linear SVM, RBF kernel SVM, and Fuzzy Logic.

\section{Results}

Modality Level Classification. We used pLDA to calculate the classification accuracy rates for each state. The results are listed in Table 2. Recall rates are shown in the table as accuracy rates. In the table, "Subject independent" means that we obtained training and test data set from different participants. "Subject dependent" means that we obtained the two kinds of data sets from the same participant. Leave one out cross validation method was used for calculating the recall rates.

Table 1 Classification Accuracy Rates for Each State by Each Modality

\begin{tabular}{|c|c|c|c|c|c|c|c|c|c|c|c|c|c|c|}
\hline \multirow[b]{3}{*}{ Modality } & \multicolumn{6}{|c|}{ Stroop Test } & \multicolumn{8}{|c|}{ Information Task } \\
\hline & \multicolumn{3}{|c|}{ Subject independent } & \multicolumn{3}{|c|}{ Subject dependent } & \multicolumn{4}{|c|}{ Subject independent } & \multicolumn{4}{|c|}{ Subject dependent } \\
\hline & Relax Concen. & Stress & All & \begin{tabular}{l|l} 
Relax & Concen.
\end{tabular} & Stress & All & Relax & Concen. & Stress & All & Relax & Concen. & Stress & All \\
\hline EDA & $76.7 \% 22.8 \%$ & $28.9 \%$ & $42.8 \%$ & $100.0 \% 97.2 \%$ & $95.6 \%$ & $97.6 \%$ & $64.4 \%$ & $10.6 \%$ & $47.8 \%$ & $40.9 \%$ & $100.0 \%$ & $97.7 \%$ & $100.0 \%$ & $99.1 \%$ \\
\hline BVP & \begin{tabular}{|l|l|}
$42.2 \%$ & $52.8 \%$ \\
\end{tabular} & $36.7 \%$ & $43.9 \%$ & \begin{tabular}{|l|l|}
$77.2 \%$ & $98.3 \%$ \\
\end{tabular} & $62.8 \%$ & $79.4 \%$ & $47.8 \%$ & $20.0 \%$ & $49.4 \%$ & $39.1 \%$ & $90.3 \%$ & $95.4 \%$ & $94.0 \%$ & $93.0 \%$ \\
\hline HR & $36.7 \% 26.7 \%$ & $50.6 \%$ & $38.0 \%$ & $88.9 \%$ & $57.8 \%$ & $76.9 \%$ & $12.8 \%$ & $38.3 \%$ & $50.0 \%$ & $33.7 \%$ & $92.6 \%$ & $63.9 \%$ & $72.7 \%$ & $75.7 \%$ \\
\hline Floor & \begin{tabular}{|l|l|}
$25.0 \%$ & $35.6 \%$ \\
\end{tabular} & $46.1 \%$ & $35.6 \%$ & \begin{tabular}{|l|l|}
$82.8 \%$ & $74.4 \%$ \\
\end{tabular} & $78.9 \%$ & $78.7 \%$ & $46.1 \%$ & $52.8 \%$ & $30.6 \%$ & $43.1 \%$ & $83.8 \%$ & $84.7 \%$ & $87.0 \%$ & $84.1 \%$ \\
\hline Backrest & $62.8 \% 31.1 \%$ & $29.4 \%$ & $41.1 \%$ & $100.0 \% 87.2 \%$ & $82.2 \%$ & $89.8 \%$ & $62.8 \%$ & $30.6 \%$ & $31.1 \%$ & $41.5 \%$ & $94.4 \%$ & $78.7 \%$ & $73.6 \%$ & $82.8 \%$ \\
\hline Seat & $38.9 \% 32.8 \%$ & $53.9 \%$ & $41.9 \%$ & $100.0 \% 99.4 \%$ & $93.9 \%$ & $97.8 \%$ & $42.2 \%$ & $27.2 \%$ & $52.8 \%$ & $40.7 \%$ & $96.8 \%$ & $87.5 \%$ & $91.2 \%$ & $92.4 \%$ \\
\hline Eye & \begin{tabular}{|l|l|}
$66.1 \%$ & $53.3 \%$ \\
\end{tabular} & $21.1 \%$ & $46.9 \%$ & \begin{tabular}{|l|l|}
$95.0 \%$ & $65.6 \%$ \\
\end{tabular} & $63.9 \%$ & $74.8 \%$ & $46.1 \%$ & $48.3 \%$ & $40.6 \%$ & $45.0 \%$ & $87.0 \%$ & $59.3 \%$ & $61.6 \%$ & $70.6 \%$ \\
\hline Phys. & \begin{tabular}{|l|l|}
$57.2 \%$ & $40.0 \%$ \\
\end{tabular} & $29.4 \%$ & $42.2 \%$ & \begin{tabular}{|l|l|}
$98.3 \%$ & $98.9 \%$ \\
\end{tabular} & $93.9 \%$ & $96.9 \%$ & $58.3 \%$ & $32.8 \%$ & $34.4 \%$ & $41.9 \%$ & $98.6 \%$ & $95.8 \%$ & $98.2 \%$ & $97.0 \%$ \\
\hline Pres. & \begin{tabular}{|l|l}
$45.6 \%$ & $58.3 \%$ \\
\end{tabular} & $46.1 \%$ & $50.0 \%$ & \begin{tabular}{|l|l|}
$99.4 \%$ & $89.4 \%$ \\
\end{tabular} & $85.6 \%$ & $90.2 \%$ & $73.9 \%$ & $55.0 \%$ & $49.4 \%$ & $57.8 \%$ & $86.6 \%$ & $81.9 \%$ & $81.5 \%$ & $91.9 \%$ \\
\hline All & \begin{tabular}{|l|l|}
$70.0 \%$ & $18.9 \%$ \\
\end{tabular} & $60.6 \%$ & $53.3 \%$ & \begin{tabular}{|l|l|}
$100.0 \%$ & $92.2 \%$ \\
\end{tabular} & $93.9 \%$ & $94.3 \%$ & $80.6 \%$ & $55.0 \%$ & $32.2 \%$ & $53.7 \%$ & $96.3 \%$ & $88.0 \%$ & $87.5 \%$ & $91.7 \%$ \\
\hline
\end{tabular}

Each column represents the two stressors, subject independent/dependent, and the three stress states. The Relax, Concen., and Stress in the table represent the relaxed, concentrated, and stressed states, respectively, and All means all the states, which is the average of the three states. Each row represents the modality. Floor, Backrest, and Seat means the pressures distribution sensor signals from each place. Eye means the signals from the eye tracker sensor. Phys. means the combined physiological signals, and Pres., the combined pressure distribution sensor signals. All means the signals from all the modality combined sensors.

The shadowed cells are for comparison between the combined pressure distribution signal, the combined physiological signal and eye movement signal. For the subject independent cases, the combined pressure sensor showed higher classification accuracy rates than the other two modalities, although this cannot be said for the subject dependent cases.

For further discussion, we also show the confusion matrix for Eye, Phys., and Pres. The shadowed cells are for comparison. The precision rates show the same tendency: 
for the subject independent cases, the combined pressure sensor showed higher classification accuracy rates than the other two modalities.

Table 2 Confusion Matrices for the three modalities

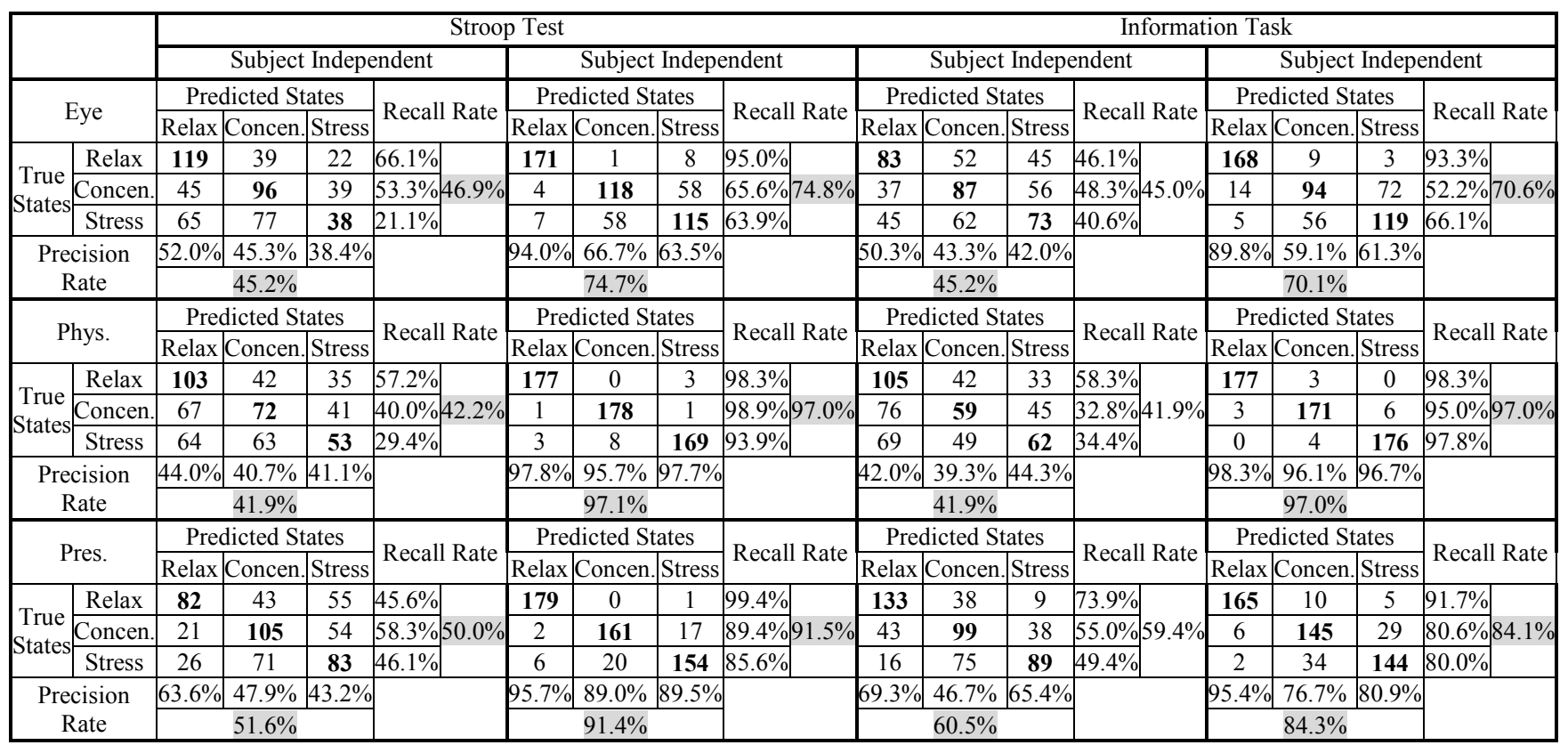

Table 3 Classification Accuracy Rates for Each State by Each Modality

\begin{tabular}{|l|l|l|l|l|l|}
\hline \multirow{2}{*}{\begin{tabular}{|l}
\multirow{2}{*}{ Classifiers } \\
\cline { 3 - 6 }
\end{tabular}} & \multicolumn{2}{c|}{$\begin{array}{c}\text { Subject } \\
\text { Independent }\end{array}$} & $\begin{array}{c}\text { Subject } \\
\text { Dependent }\end{array}$ & $\begin{array}{c}\text { Subject } \\
\text { Independent }\end{array}$ & $\begin{array}{c}\text { Subject } \\
\text { Dependent }\end{array}$ \\
\hline \multirow{3}{*}{$\begin{array}{l}\text { Decision } \\
\text { Level } \\
\text { Fusion }\end{array}$} & $\mathrm{kNN}$ & $45.74 \%$ & $81.67 \%$ & $49.81 \%$ & $90.93 \%$ \\
\cline { 2 - 6 } & pLDA & $48.89 \%$ & $85.74 \%$ & $51.48 \%$ & $92.96 \%$ \\
\cline { 2 - 6 } & Linear SVM & $46.48 \%$ & $84.07 \%$ & $49.81 \%$ & $93.15 \%$ \\
\cline { 2 - 6 } & RBF SVM & $45.56 \%$ & $84.26 \%$ & $49.26 \%$ & $\mathbf{9 3 . 5 2 \%}$ \\
\cline { 2 - 6 } & Fuzzy Logic & $45.93 \%$ & $80.00 \%$ & $\mathbf{5 4 . 2 6 \%}$ & $87.59 \%$ \\
\hline Feature Level Fusion & $\mathbf{5 3 . 3 3 \%}$ & $\mathbf{9 4 . 2 6 \%}$ & $53.70 \%$ & $91.67 \%$ \\
\hline
\end{tabular}

Decision Level Classification. With the decision level fusion method using decision level features shown equation (1), we classified the three stress states using several classifiers for the four cases. The results are summarized in Table 3. Again, recall rates are shown in the table as accuracy rates. The feature level fusion results, using pLDA as the classifier, are also itemized in the table as reference.

For the information task states, our decision level fusion method worked well, showing higher rates than those of the feature level fusion. However, for the Stroop test states, our method could not obtain higher rates compared with the feature level fusion. 


\section{Discussions and Conclusion}

Multi-modality is an effective method to recognize stress or emotion. In the state of the art of stress recognition, many modalities are used. However, the accuracy rates of each modality for each stress state have not yet been systematically tested and compared with each other, though this is important because it will provide the information for selecting and combining modalities to predict each state.

Therefore, the accuracy rates listed in Table 1, providing insights on the contribution of each single modality to stress state recognition, are important. Among them, one of the most remarkable results is that the pressure combined signal had higher prediction accuracy than the physiological combined signals or eye tracker signals in the subject independent case, for the two stressors (Stroop and Information Task) on average (All state). On the one hand, this is surprising because these two modalities (physiological signals and eye movement signals) are considered as involuntary modality and directly reflect neural activities which include emotion and stress. On the other hand, postures can be controlled voluntary. We will conduct further research to confirm the tendency shown in this research, and find the reason to underline the importance of using pressure distribution sensors. The confirmation of this result will make a new step towards the possibility of the usefulness of body placement for stress recognition in daily work, especially for unobtrusive, user independent systems.

The accuracy rates listed in Table 1 can be used not only for the selection or combination of modalities, but also for the fusion of the 'decision' of modalities to predict stress states. We demonstrated a decision level fusion; however, the result was not desirable. We will develop more advanced decision level fusion methods to take advantage of this information, especially for the subject independent case.

\section{References}

1. Demerouti, E., Bakker, A. B., Nachreiner, F., Schaufeli, W. B: The job demands-resources model of burnout. J. Appl. Psychol. 86(3), 499-512(2001)

2. Grafsgaard, J.F., Boyer, K.E., Wiebe, E.N., Lester, J.C.: Analyzing Posture and Affect in Task-Oriented Tutoring. $25^{\text {th }}$ International Florida Artificial Intelligence Research Society Conference, 2012

3. Lang, P.J., Bradley, M.M., Cuthbert, B.N.: Emotion, Motivation, and Anxiety: Brain Mechanisms and Psychophysiology. Biol. Psychiatry 44(12), 1248-1263(1998)

4. Arnrich, B., Setz, C., La Marca, R., Tröster, G., Ehlert, U.: What Does Your Chair Know About Your Stress Level? IEEE Trans. Inf. Technol. Biomed. 14(2), 207-214(2009)

5. Frank, K., Robertson, P., Gross M., Wiesner, K. :Sensor-based Identification of Human Stress Levels. International Conference on Pervasive Computing and Communications Workshops, pp.127-132(2013)

6. Calibo, T.K., Blanco, J.A., Firebaugh, S. L.: Cognitive Stress Recognition. Instrumentation and Measurement Technology Conference, pp.1471-1474(2013)

7. Meyer, J., Arnrich, B., Schumm J., Tröster, G.: Design and Modeling of a Textile Pressure Sensor for Sitting Posture Classification. IEEE Sensor Journal. 10(8), 1391-1398( 2010) 
8. Hernandez, J., Paredes, P., Roseway, A., Czerwinski, M.,: Under Pressure: Sensing Stress of Computer Users. SIGCHI Conference on Human Factors in Computing Systems, pp.51-60(2014)

9. Hart, S.G., Staveland, L.E.: Development of NASA-TLX (Task Load Index): Results of Empirical and Theoretical Research. Adv. Psychol. 52, 139-183(1998)

10. Giraud, T., Soury, M., Hua, J., Delaborde, A., Tahon, M., Antonio, D., Jauregui, G., Eyharabide, V., Filaire, E., Le Scanff, C., Devillers, L., Isableu B., Martin, J.C., : Multimodal Expressions of Stress during a Public Speaking Task. 5th Biannual Conference of the Humaine-Association on Affective Computing and Intelligent Interaction, pp.417-422(2013)

11. Plarre, K., Raij, A., Hossain, S.M., Ahsan Ali, A., Nakajima, M., al'Absi, M., Ertin, E., Kamarck, T., Kumar, S., Scott, M., Siewiorek, D., Smailagic, A., Wittmers Jr., L. E.: Continuous Inference of Psychological Stress from Sensory Measurements Collected in the Natural Environment. 10 ${ }^{\text {th }}$ International Conference of Information Processing in Sensor Networks, pp.97-108(2011)

12. Renaud, P., Blondin, J.P.: The stress of Stroop performance: physiological and emotional responses to color word interference, task pacing, and pacing speed. Int. J. Psychophysiol. 27(2), 87-92(1997)

13. Zhai, J., Barreto, A.: Stress Detection in Computer Users Based on Digital Signal Processing of Noninvasive Physiological Variables," $28^{\text {th }}$ Annual Conference of IEEE Engineering in Medicine and Biology Society, Vols 1-15, pp.1999-2002(2006)

14. Di Stasi,L.L., Catenad, A.,Cãnasc,J.J, Macknike, S.L., Martinez-Condea, S.: Saccadic velocity as an arousal index in naturalistic tasks. Neurosci. Biobehav. Rev. 37(5), 968-975(2013)

15. SensingTex, http://sensingtex.com/

16. Wild Divine, http://www.wilddivine.com/

17. TheEyeTribe, https://theeyetribe.com/

18. Wagner, J., Lingenfelser, F., André, E.: The Social Signal Interpretation Framework (SSI) for Real Time Signal Processing and Recognition. $12^{\text {th }}$ Annual Conference of the International Speech Communication Association. Vols.1-5, pp.3252-3255( 2011)

19. Jackson, M.D., McClelland, J. L.: Sensory and Cognitive Determinants of Reading Speed. J. Verbal Learning Verbal Behav. 14(6), 565-574(1975)

20. Picard, R.W., Vyzas, E., Healey, J.: Toward Machine Emotional Intelligence: Analysis of Affective Physiological State. IEEE Trans. Pattern Anal. Mach. Intell. 23(10), 1175-1191 (2001)

21. Peterka, R.J., Loughlin, P.J.: Dynamic Regulation of Sensorimotor Integration in Human Postural Control. Journal of Neurophysiology 91(1), 410-423(2004)

22. Krafczyk, S., Schlamp, V., Dieterich, M., Haberhauer, P., Brandt, T.: Increased body sway at 3.5-8 Hz in patients with phobic postural vertigo. Neurosci.Lett. 259(3), 149-152(1998)

23. Nodine, C.F., Kundel, H.L., Toto, L.C., Krupinski, E.A.: Recording and analyzing eye-position data using a microcomputer workstation," Behav. Res. Methods. Instrum. Comput. 24(3), 475-485(1992)

24. Manor, B.R., Gordon, E.,: Defining the temporal threshold for ocular fixation in free-viewing visuocognitive tasks. J. Neurosci. Methods. 128(1-2), 85-93(2003)

25. Zhai, J., Barreto, A.B., Chin, C., Li, C.: Realization of Stress Detection Using Psychophysiological Signals for Improvement of Human-Computer Interaction," Proceedings of IEEE SoutheastCon, pp.415-420(2005)

26. Mokhayeri, F., Akbarzadeh, M.R., Toosizadeh, T, S.: Mental Stress Detection Using Physiological Signals Based on Soft Computing Techniques, $18^{\text {th }}$ Iranian Conference of Biomedical Engineering (ICBME), pp. 232-237( 2011)

27. Koldijk, S., Sappelli, M., Verberne, S., Neerincx, M.A., Kraaij, W.: The SWELL Knowledge Work Dataset for Stress and User Modeling Research," $16^{\text {th }}$ International Conference of Multimodal Interaction, pp.291-298(2014)

28. Kim, J.: Bimodal Emotion Recognition using Speech and Physiological Changes. Robust Speech Recognition and Understanding, Grimm, M., Kroschel,K.,(eds.), I-Tech Education and Publishing, pp. 265- 280(2007) 